\title{
Innovation Study of Visual Thinking Mode in Visual Communication Design
}

\author{
${ }^{1}$ Zhang Li, Su Wen \\ Department Of Art and Design, GuangDong University Of Science \&Technology \\ Nancheng District, Dongguan, Guangdong
}

\begin{abstract}
Visual thinking and visual communication design are closely linked, and the two are somewhat compatible. Visual communication design requires the use of visual thinking patterns and regards it as an important support. Therefore, the innovation of visual thinking mode can effectively improve the level and quality of visual communication design. Based on this, this paper studies the innovation of visual thinking mode in visual communication design.
\end{abstract}

Keywords-Visual thinking mode; Visual communication design; Innovation study

\section{INTRODUCTION}

Vision is an instinctive perceptual intuition to acquire and receive information, and thinking is the process of analyzing, screening, judging and reasoning the acquired information. In the past, people have separated the study of vision and thinking. Nowadays, many domestic and foreign scholars have proposed the concept of visual thinking, that is, the visual image formed by the brain will be converted under the premise that the eye responds normally to external stimuli. Analytical material for visual thinking. Visual communication design mainly refers to the design process of information transmission by means of visual symbols. In the design process, both the audience and the designer themselves need to transmit information according to visual symbols, which is inseparable from visual thinking.

Visual communication design is a design method, but it is not limited to design means. It is also a kind of design method, and it is also the scope of design concept. Visual communication design continues to advance with the development of science and technology. It is not only affected by the visual image of the recipient, but also needs to display its own characteristics when completing the visual communication design. In recent years, visual communication design has begun to change from static to dynamic, and its expression has gradually changed from the previous twodimensional picture to the three-dimensional picture or fourdimensional space-time picture. At this time, the application of innovative thinking is very much needed. Only by continuous innovation and research in the works can the design reach a state of advancing with the times, and it can bring the audience a bright feeling. The innovative thinking in visual communication design is a comprehensive innovation, which is a comprehensive innovation, which includes all aspects of form, content, and thought. It is a transcendence of traditional art and an exploration of future art.
Innovative thinking is a new way of brain activity, and its classification can make its development more clear and its application of the model more flexible. First, the divergent thinking mode. It means that the brain will present a state of diffusion when thinking, which allows the brain to produce extremely rich associations and expand the breadth of thinking. On the one hand, this mode of thinking can think of another problem through one problem. On the other hand, it can also think about the same problem from different angles. Second, the reverse thinking mode, which is contrary to the traditional mode of thinking, often Starting with the most common things or viewpoints, use reverse thinking to study the problem. Often able to bring people a new creative experience; Third, the associative thinking mode, this mode of thinking is a free-style thinking activity that is caused by the different appearances caused by certain incentives generated in the brain. It is very common in our lives and is a way of thinking that designers can generate ideas.

Visual communication design is not only a design method, but also a design method, and also belongs to the scope of design concepts. In today's society where technology is rapidly developing, visual communication design should be based on the visual image of the recipient. This requires that the completed visual communication design should have humanized characteristics to resonate with the recipient. In addition, visual communication design works have gradually shifted from static communication expressions to dynamic communication expressions. The visual communication design has changed the previous two-dimensional plane space, and has been extended to three-dimensional and even four-dimensional space-time space. At the same time, the future of visual communication design thinking will develop into a new trend of cross-cutting and interactive. Innovation is the survival foundation of the design industry. Only by persisting in innovation can the visual communication design continue to evolve in line with the requirements of the times. The innovation to realize visual communication design is a comprehensive and comprehensive innovation. It is not only the artistic form and ideological content of innovative visual communication design works, but also the change of traditional artistic concept. It is a transcendence realization of the past art. 


\section{VISUAL THINKING PATTERNS IN VISUAL COMMUNICATION DESIGN}

On the basis of the normal response to external stimuli, the visual image is formed in the brain and becomes a direct material for visual thinking. Visual thinking refers to the process of analyzing, summarizing, processing, organizing, and understanding the meaning of the perceived visual image to obtain the information it wants to express. Visual thinking is a kind of rational thinking movement. It is a creative understanding and grasp of the perceived things. It relies on visual images to observe and imagine the interactive operation to continuously construct the cognitive activities. The visual thinking is different from the general logical thinking, and presents its unique characteristics of non-linguistic, creative, intuitive, divergent, emotional, and comprehensive.

There are three modes of thinking in visual communication design: 1 divergent thinking mode, which refers to the brain's thinking state of diverging the accepted information. It not only makes the thinking more broad, but also can be directed at different angles of the problem. Comprehensive comparison and association. 2 Reverse thinking mode refers to the thinking mode of thinking backwards about some seemingly ordinary things or viewpoints. Reverse thinking allows designers to break the rules and create creative designs. 3 Associative thinking: refers to a spontaneous and free-thinking movement of different images and brains due to a chain reaction of some internal incentives. Scientifically using associative thinking, designers can combine different images with appropriate adjustments through association and imagination, thus taking into account a new creative design ideas and innovative creations.

Where the idea comes from, sometimes it takes a lot of hard work, and sometimes it suddenly appears as a flash of light. In fact, visual thinking plays a role that cannot be ignored. Designers create creative thinking by visually thinking about visual images. Under the ever-changing science and technology background, the visual communication design has also made corresponding progress. The development trend is influenced by the subjective visual image of the recipient. Using visual imagery to filter the creativity can make the viewer at the first sight Responding to it has also become one of the criteria for judging creativity. Therefore, designers should flexibly apply visual thinking patterns to comprehensively innovate from all aspects of visual imagery, thus more in line with people's needs for design.

\section{INNOVATIVE PRINCIPLES OF VISUAL THINKING IN VISUAL COMMUNICATION DESIGN}

Visual thinking has obvious characteristics of interest in the process of searching for images. People only scan the images that they care about or are interested in. Once the visual cognition results, they quickly move to another center of interest. Form the overall process of visual thinking. This process of visual thinking effectively guarantees the speed and possibility of object recognition, but it is neglected and ambiguous to images outside the visual center, so it is necessary to pay attention to the visual center.
Proportion is both a measure and a description of beauty, and it also explains the important role that scale plays for the beauty itself. The relationship between scale and scale mainly refers to the relationship between the whole and the part of the thing itself, and there is a certain proportion of contrast between them, which is also a kind of harmonious embodiment The scale and proportion are very different, which are the main factors that directly affect people's aesthetic judgment and psychological feelings. The degree of grasp of it can truly reflect the level of self-cultivation and level of the designers themselves.

Visual thinking is an overall impression and complete feeling of the physical aspect. It is a creative process to organize and improve its own cognitive objects. In the process of recognizing the characteristics of the object, visual thinking can be consciously grouped according to a variety of different elements, and can be classified and identified according to the tension of its similar factors, shape, line and space. On the basis of this perception, visual thinking can consciously supplement the corresponding image part, thus promoting its image consciousness of integrity in the heart.

\section{THE INNOVATION OF VISUAL THINKING MODE IN VISUAL DESIGN}

Emphasize focus on vision. In the process of running visual thinking to search all the images in the mind, there is a remarkable feature that only interested in the most important and meaningful parts in the process of browsing the works, that is, there is a concern, only concerned with being able to The existing image in the brain responds to the picture and ignores the unfamiliar and unfamiliar picture. The visual center is in a prominent position in people's visual perception, which affects people's judgment and understanding of design concepts. Therefore, in the visual communication design, we must emphasize the visual center, so that people can have an interest in your work, in order to understand the content and connotation of the design itself.

Grasp the scale and scale. In ancient times, people like to use "one point is too fat, one point is too thin" to praise the beauty, which shows that the importance of the proportion to the United States, can be described as a loss of a thousand miles, a thousand miles. Proportions and scales exist in the whole and part of things, and describe the harmonious and well-balanced charms displayed by certain contrasts. The perfect proportion, the appropriate scale difference is the basis of structural beauty.

Control comparison and unification. The difference between the two effects is the contrast. In terms of the effect of expression and expression, contrast has its unique artistic effect and value. It is also a means of visual communication design. For example, the shade of the hue, the warmth and the warmth, the vertical and horizontal direction, the number of the number, the density of the arrangement, the up and down, the left and right positions, the shape of the real, the light and heavy, etc., can be used in the design. And unification is a main method of regularly arranging the overall situation of planning, so as to grasp certain rules and obtain overall aesthetics and intentions. 
Control of symmetry and balance. Symmetrical things can produce a natural, harmonious, elegant, and solemn visual aesthetic, and it also adapts to people's sensory habits. Therefore, designers should effectively use this image thinking according to the consensus of people's aesthetics. Equilibrium is an asymmetrical form that is not the same as symmetry. Symmetry is absolute, and equilibrium is a stable form of coordinating people's senses and psychology.

Coordination with the whole and the part. Visual thinking is the overall feeling and impression of the object being known, and can organize and perfect the perceived object. Only by comprehensively understanding and recognizing things can we continuously improve the design concept, design better works, and constantly improve the quality of design. Grasping the whole and part can be divided according to each element when recognizing things, and it can relax and express each element and classify and identify the dotted line. Visual thinking completes a complete image in the heart by grasping the overall perception and locality.

1) Use divergent thinking. For a long time, divergent thinking has been widely recognized as a creative thinking. Divergent thinking is a special mode of thinking when designers design, and it is the application of visual thinking mode in visual communication design. Designers need to consider many factors when designing. When designing, this requires designers to consider the imagination from a variety of perspectives, and combine the purpose and concept of the design work to add richer connotation and meaning to the design. To make it more appealing. However, when using divergent thinking, it should be reasonably diverged according to the design's intentions and requirements. 2) Apply reverse thinking. In the design of visual communication, reverse thinking can bring people a new sensory experience. If a designer uses reverse thinking to create a design, he is likely to obtain unique creative effects. In the design process, by thinking about all aspects of the object, you can enrich the connotation of the work and achieve unexpected creative effects. At the same time, when collecting design materials, designers can pay attention to creative or special cases, and use different materials in the design process to show different effects and deepen people's impression of the works. Especially when designers can hardly express the information they want to express according to the conventional visual thinking mode, by using reverse thinking and thinking and integrating the materials existing in the mind, they can open a paradise of inspiration. 3) Apply associative thinking. Associative thinking is widely used in visual communication design and is a common way of thinking. In the design process, the designer associates something with other things, and through this process of thinking, a new thinking idea is created, which can improve the quality of the work and better convey the design meaning and meaning. Lenovo has three types: Lenovo, Comparative Lenovo, and Causal Association. Any of these forms of association is a way of thinking that visual designers often use when creating. According to the specific situation, the designer selects the appropriate thinking mode, better designs and injects new Lenovo ideas. 4) Update the design concept with the times. With the continuous development and progress of the society, innovation has become a trend in the development of various fields. Visual communication designers need to update their design concepts and keep pace with the times so as to better adapt to the development of the times. Designers should constantly learn and visit to enhance their artistic accomplishment and aesthetics. The design concept will change as people's aesthetic concepts change. Only by keeping pace with the development of the times, the designed works can better reflect the cultural characteristics of the times and are more likely to cause the public to understand and love. 5) Apply innovative visual language. Language is one of the expression tools, and visual communication designers also have special design terms when designing. The design term fully expresses the information that the artwork requires to be passed on to people. The visual art language of visual communication design mainly includes elements such as styling, text, color, etc. The scientific combination of the above elements can complete excellent visual design works. Since artistic language is a kind of expressive expression in visual communication design, people can first perceive that the recognized information needs to convey information from the visual art language. Therefore, the use of innovative artistic language is closely related to the innovation of the mode of visual thinking. 6) Apply science and technology. Nowadays, we are in the era of science and technology information that the network is deeply rooted in the ordinary people's life and multimedia is popularized by the masses. Designers can make full use of the advantages of the modern era to create, diversify the design and enrich its connotation. Computers have long been used as an auxiliary tool by ordinary people because of their convenience, proficiency and strong advantages. Among them, designers are even more engaged. Under the promotion of advanced technologies such as multimedia and network, advanced technology can also be used. To the extent that the designer's work efficiency and work quality are improved, the digitalization of visual communication design has become an inevitable development trend, and at the same time, it has directly accelerated the rapid development of the entire visual communication design industry in China. 7) Conduct inductive creative thinking training. Inspiration is the core of design, the soul of design, just like CPU and computer. When a designer lacks inspiration, it can also be inspired by intentional inspiration, re-inspired and creatively designed to achieve innovative meaning. works. The visual communication designer can find the essence and characteristics of the visual image according to the design requirements of the design work. Through the artificial selection of various elements, grasp the design concept and theme, search for relevant materials, get inspiration from various channels, and thus better. Design creation. 


\section{EFFECTIVE STRATEGIES FOR INNOVATIVE VISUAL THINKING IN VISUAL COMMUNICATION DESIGN}

In the visual communication design, the visual thinking should be continuously improved and applied, so that the visual innovative thinking can better serve the visual communication design, enriching its expression and enhancing its expressive power on the basis of ensuring the visual communication design concept. Let the audience feel the new ideas from the rich design and feel the shock of the design.

Divergent thinking is often mentioned in our lives, but in theory it is more commonly used by psychologists. From a psychological perspective, divergent thinking is a very important application and characteristic in creative thinking. A very effective way to enhance the visual designer's innovative thinking is to train his divergent thinking. For example, for the same thing, let the designers carry out the idea of "smart", and look for the inspiration of visual communication design from these ideas. Training in this way can make the designer's divergent thinking mode more active, which can expand the designer's design ideas and help his innovative thinking.

Reverse thinking can bring a new feeling to the audience. Therefore, training the designer's reverse thinking is also an effective way to expand the innovative thinking mode. On the one hand, designers should consciously train their own reverse thinking, designing things through a certain thing from the back to the front, or from the bottom up, so that they can incorporate different design creative effects in the design; On the other hand, designers can also carry out reverse thinking training from the collection of materials, through the collection of special materials to achieve the creative effect of the design, so that the design works produce unexpected design effects. Reverse thinking is often different from people's normal thinking, and it is this difference that makes the work creative and fresh, which makes people impressed with the design.

Associative thinking is no stranger to our lives. It can also be applied to visual communication design, connecting the association of a thing through a thing to achieve the generation of an innovative thinking. Lenovo is mainly divided into contrasting associations, proximity associations, and causal associations. Any of these association methods can be used in visual communication design works to enhance the quality and creativity of the work. When the designer conducts the association thinking training, he can start from the life, associate the relevant content and materials, and record it to understand his own association trajectory, and find the best match point with the work, so as to design the work. Flexible application in the middle. Associative thinking exists in every corner of our lives. Designers must have a pair of eyes that are good at discovering, and those who are diligent in thinking, so that associative thinking can take root in the body. Innovation is the source of any work development and progress, the essential requirement for the improvement of work quality, and the important standard given by the times. The same is true for visual communication design. Designers must put forward strict requirements for themselves, constantly research the content related to the works, exercise the innovative thinking, keep the brain running at a high speed, and give full play to their subjective initiative.

\section{INNOVATIVE THINKING OF VISUAL THINKING IN VISUAL COMMUNICATION DESIGN}

This mode of thinking is also called diversified thinking, mainly a mode of thinking that has become a conclusive view or thing from the opposite direction. This mode of thinking guides the designer to think backwards and easily stand out from the conventional design philosophy to design creative designs. Therefore, in visual communication design, designers can adopt this kind of reverse thinking to break the routine and make their ideas more active and more creative, so as to achieve unexpected artistic effects. The associative thinking mode mainly refers to some kind of dynamic thinking activity in the brain that generates different patterns that are related to each other because of different inducing factors. This kind of associative thinking is closer to various more abstract association models due to the different nature of objects, which means that the effective association between many different things is directly caused by the closeness of time and space. Lenovo is a calm, rational and logical mindset, and it is also the most common mode of use in daily life and work. The visual communication design uses the associative thinking to inject new content into the original attributes of the work, thus creating a new creative thinking.

Divergent thinking is a diffuse state of mind in the process of thinking. In this state of thinking, the thinking horizon of thinking is usually very broad, and generally presents a multidimensional divergence state. Using this divergent way of thinking can be a comprehensive reflection of the problem from multiple angles, so it is open and diverse. Moreover, the biggest feature of divergent thinking is that it is very creative. Therefore, using divergent thinking in the visual communication design process, we can find the best and most innovative solutions in many directions of thinking, so we design for visual communication. Provide a rich source of creativity and inspiration.

\section{CONCLUSION}

Innovation is the requirement of the development of the times. Designers should strengthen the training of innovative thinking, enrich their experience, enrich the connotation of visual thinking, keep up with the pace of the times, create works that conform to people's aesthetic concepts, and form their own artistic style through innovative visual thinking patterns. To ensure the artistic effect of the work. 


\section{ACKNOWLEDGMENT}

Guang Dong University Of Science \&Technology Hospital level project: <Dongguan intangible cultural heritage "thousand corner lamp" skills into the visual communication design professional teaching practice research>.Project number: CQ2016042.

Guang Dong University Of Science \&Technology Hospital level project: <Research on the current situation and trend of visual communication design in Dongguan>. Project number: GKY-2017KYQN-20.

Guang Dong University Of Science \&Technology Hospital level project: <Research on the value and development trend of Dongguan soft decoration design industry>. Project number: GKY-2017KYQN-21.

\section{REFERENCES}

[1] Cao Wenqi. Innovation of Visual Thinking Mode in Visual Communication Design [J]. Jiangxi Social Sciences, 2012(8): 216-219.

[2] Feng He. Research on Visual Thinking Model Innovation Based on Visual Communication Design [J]. Journal of Chizhou University, 2015, 29(5): 116-118.

[3] Cao Wenqi. Innovation in visual thinking mode in visual communication design [J]. Jiangxi Social Sciences, 2012 (8): 216 - 219.

[4] Wang Qiuqiu. Innovation in visual thinking mode in visual communication design [J]. Northern Literature (late issue), 2016(3): 225 -225 .

[5] Liu Yujun. Innovation in visual thinking mode in visual communication design [J]. Architectural Engineering Technology and Design, 2016(33): 1788 . 